\title{
Distributed fibre optic sensor system to measure the progressive axial shortening of a high-rise building during construction
}

\author{
Nicholas de Battista \\ University of Cambridge, Cambridge, U.K. \\ Epsimon Ltd., U.K.
}

Ross Harvey

WSP Group, London, U.K.

Nick Cheal

Multiplex Construction Europe Ltd., London, U.K.

Contact: $\underline{\text { n.debattista1@gmail.com }}$

\begin{abstract}
A novel approach is being used to measure the progressive axial shortening of key structural elements of Principal Tower, a 50-storey reinforced concrete building in London, as it is being built. Distributed fibre optic sensor (DFOS) cables are embedded inside two columns and two core walls, from which the axial strain profile can be measured along the whole height of the constructed elements. Measurements are being taken regularly throughout the construction process, making it possible to observe the change in strain, and thus the axial shortening, within these elements, at any stage of the construction. This helps the design engineers and contractor verify the predicted differential shortening and adjust the column height presets if necessary. The purpose of this paper is to describe the monitoring system and to present initial data recorded from the first five levels of the building.
\end{abstract}

Keywords: axial shortening; differential shortening; high-rise; tall buildings; jumpform; monitoring; distributed fibre optic sensors; Brillouin sensing; Brillouin optical time domain analysis (BOTDA).

\section{Introduction}

Axial shortening of vertical load-bearing elements is a critical parameter in the design and construction of reinforced concrete high-rise buildings. Differences between the shortening of adjacent columns, and between the shortening of columns and shear core walls, give rise to additional forces in the connecting floor slabs and beams [1], which need to be catered for in the structural design. Differential shortening can also damage non-structural elements such as finishes, external cladding, mechanical services and partition walls, if these are not installed with sufficient tolerance and inbuilt flexibility [2]. Therefore, it is important to have an a-priori understanding of the long-term site-specific behaviour of the structural members, in order to 
inform the column pre-setting and the fit-out of the building.

On the other hand, the time-dependent shortening of columns and walls is notoriously difficult to predict at the design stage. Several methods have been proposed to assist engineers in estimating the elastic (due to superimposed load) and inelastic (due to creep and shrinkage) shortening of individual structural members [1-3], and to compensate for the expected differential shortening [4, 5]. In addition, one also has to consider the deformation due to environmental effects. The difficulty lies in the fact that the axial shortening of reinforced concrete members depends on the interaction of several parameters. Some of these are known or can be estimated fairly accurately (such as concrete strength, steel reinforcement and member stiffness and shape) while others are unpredictable and tend to change with time (such as relative humidity, ambient temperature and direct solar radiation).

Faced with this uncertainty concerning such a critical design parameter, the only recourse for peace of mind is to measure the actual shortening of the columns and walls during construction and, if possible, during the lifetime of the building. However, due to the difficulty of installing and maintaining sensors, instrumentation of high-rise buildings to measure axial shortening is far from systematic. Some examples of such monitoring include the use of embedded vibrating wire strain gauges (VWSGs) at six levels of a 69-storey building [6]; VWSG-based wireless sensor nodes at two levels of a 66-storey and two levels of a 72storey building [7]; and fibre optic long-gauge strain sensors (SOFO) in the ground level of ten columns of a 19-storey building [8].

Instrumentation used thus far to measure axial shortening of buildings has consisted of point sensors, which can only be used to measure the deformation at the specific level where they are installed. This is a crucial limitation since they cannot provide a measure either of the local member shortening at un-instrumented floors, or of the global shortening of the whole building. In addition, each point sensor requires its own cabled (in the case of wired sensors) or wireless radio (in the case of wireless sensor nodes) connection to a data logger. Due to financial and practical constraints, point sensor systems are invariably limited to a few tens of monitoring points per building at most.

To overcome these limitations, a monitoring system consisting of distributed fibre optic sensors (DFOS) is proposed and installed for the first time to measure the axial deformation at Principal Tower, a 50-storey reinforced concrete building being constructed in London. The continuous fibre optic sensing cables are embedded within two columns and two shear core walls, for the full height of the building, as it is being constructed. At the same time, a Brillouin optical time domain analysis (BOTDA) spectrum analyser is used to measure the strain and temperature along the embedded FO cables. This allows the engineers and contractor to derive the actual axial deformation along the entire instrumented members, at any point in time.

\section{Design and construction of Principal Tower}

Principal Tower is a residential development designed by Foster + Partners and constructed by Multiplex (Figure 2.1). It is situated between the City and Shoreditch areas in central London. The

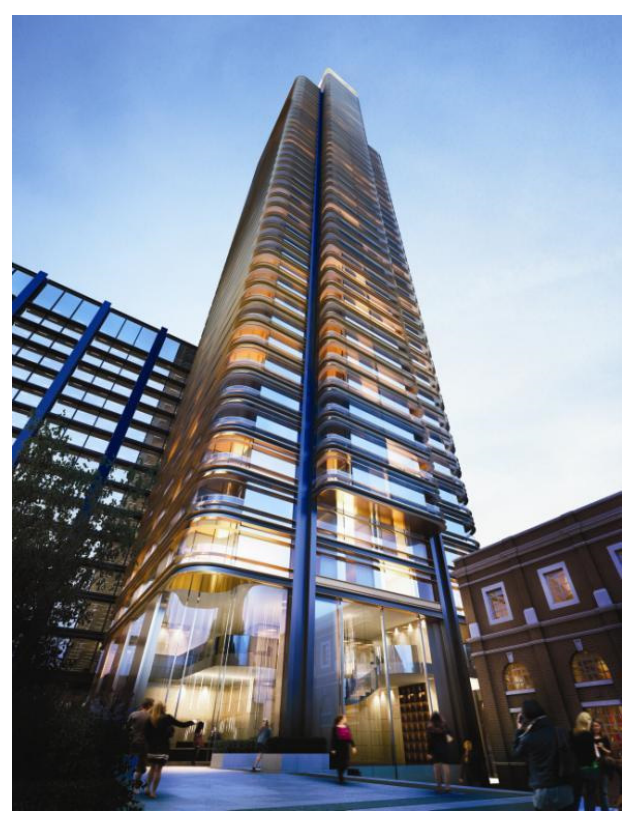

Figure 2.1. Illustration showing the completed Principal Place Tower. 
$163 \mathrm{~m}$-tall building consists of 50 storeys above ground. Except for a $5 \mathrm{~m}$-high entrance foyer at ground floor (level 0 ), each level is approximately $3 \mathrm{~m}$ high. There are also two basement levels under the tower.

At ground level, the tower has a footprint of approximately $640 \mathrm{~m}^{2}$ and part of it spans over the route of the future railway tunnel out of Liverpool Street Station. This required complex load transfer systems to be designed and constructed in the building's lower levels.

\subsection{Structural system}

The structural system of the tower was designed by WSP. It comprises a reinforced concrete core providing stability and encapsulating the lifts, 200-225 mm-thick post-tensioned floor slabs, and 12 columns positioned around the perimeter of the building (Figure 2.2). Concrete grade C60/75 was used for columns and walls, and C40/50 was used for slabs.

The columns that coincide with the railway tunnel alignment beneath, rest on vibration-isolating rubber bearings and are supported by steel transfer beams. Double-storey outrigger walls are situated in apartment demise lines, at regular intervals along the building's height, providing the required sway resistance to wind loads.

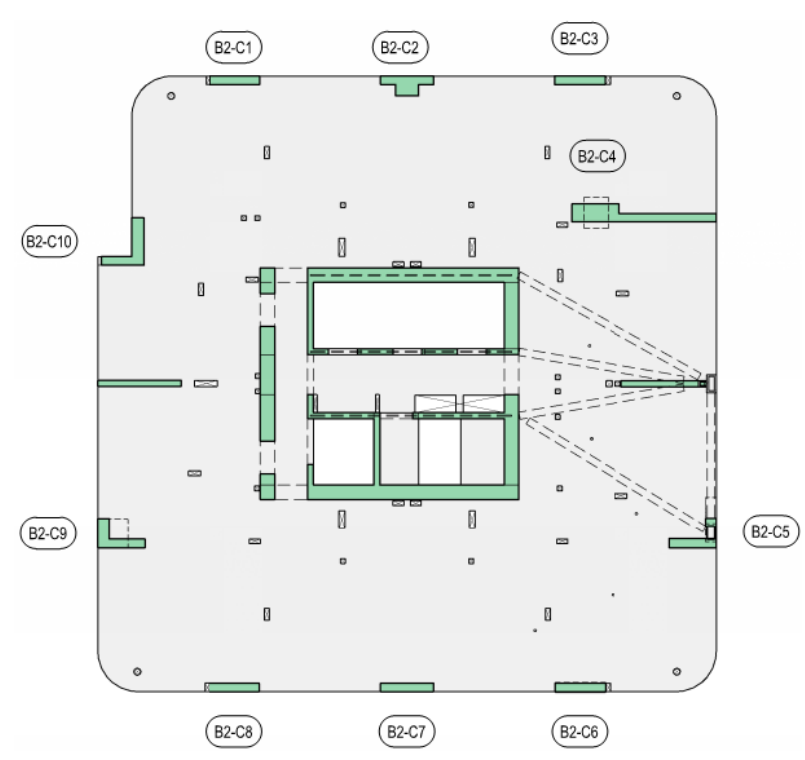

Figure 2.2. Typical floor plan of Principal Tower.

\subsection{Construction technique}

The basement levels beneath the tower were constructed using a top-down method. This allowed construction works on the tower's superstructure to start as soon as the piling and temporary ground support works were completed. An automated jumpform system was chosen as it allowed a rapid construction program to be achieved, without compromising on quality. This system is not dependent on cranes, as it uses an array of 20 tonne-capacity jacks that lift the rig off the newly cast concrete in the walls and columns. The jacks are then retracted to allow work on the next level to proceed.

For the first nine levels, the core was erected using the jumpform (Figure 2.3), while the slabs and columns were formed using traditional falsework and shuttering. From the tenth level, the jumpform rig was adapted and expanded to incorporate the whole floor footprint, such that all the vertical elements can be constructed using the jumpform system. This is the first building in the UK to use such a construction technique. Construction of the tower structure started in July 2016 and, at the time of writing (February 2017), the tenth level had been completed.

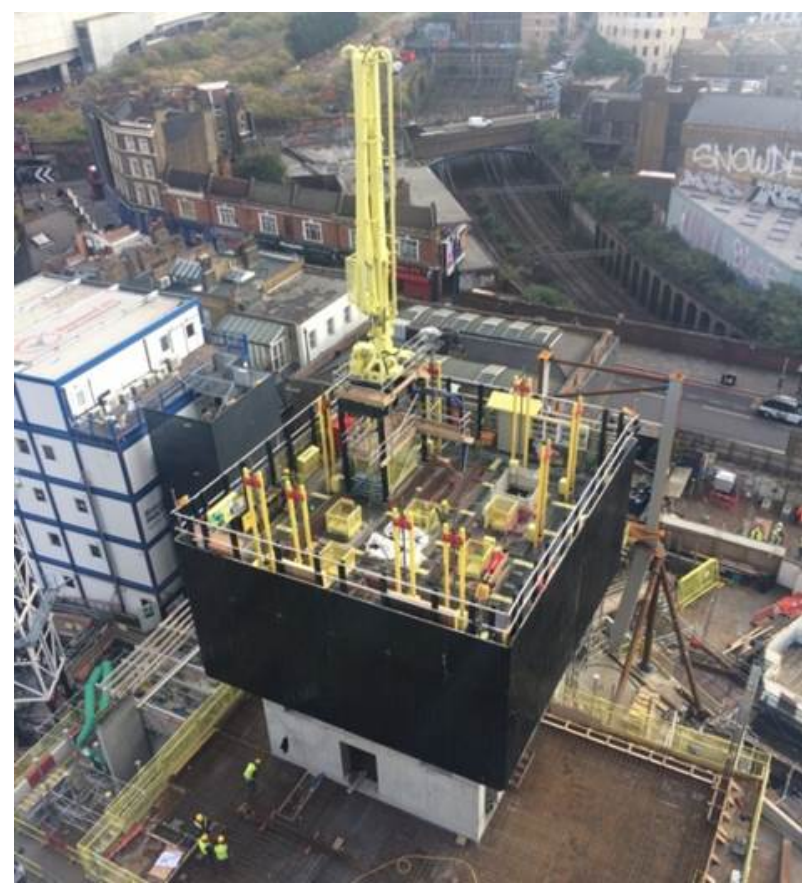

Figure 2.3. The lower levels of the core being constructed using an automated jumpform. 


\subsection{Axial shortening estimation}

In order to estimate the axial shortening of the core walls and of each column individually, WSP undertook a construction stage analysis. This accounted for the time-dependent change in the material properties of concrete and the application of load as the building is constructed. A concrete shrinkage coefficient of $242 \mu \mathrm{m} / \mathrm{m}$ was used, as derived from shrinkage testing of the concrete mix designed for the columns and walls.

An additional complication at Principal Tower was the expected settlement of the columns supported by the load transfer system. Although this does not affect the axial shortening of the columns, the height presets for the installation of the cladding and internal partitions required accurate prediction of the combined vertical movement due to settlement and shortening.

The façade cladding and fit-out works program was planned to overlap the construction program, with a lag of 10 storeys. Therefore, it was imperative that the floor-to-floor height presets were estimated accurately, taking into account the future column and wall shortening when the floors above were constructed. In order to verify these estimates and adjust them in time if necessary, a monitoring system to measure the axial shortening was required. The project engineers and contractor engaged the Cambridge Centre for Smart Infrastructure and Construction (CSIC) to install an embedded monitoring system using DFOS.

\section{Distributed fibre optic monitoring system to measure axial shortening}

Two adjacent columns ( $\mathrm{C} 8$ and $\mathrm{C} 9$ ) and two core walls (W1 and W2) were chosen to be monitored (Figure 3.1). The choice of monitoring location was based on the column profiles and heights, and on the expected shortening of the members. All four instrumented elements are supported directly by the foundations beneath the basement levels and not by the transfer structure.

Column C8 has a rectangular profile of $2.1 \times 0.35 \mathrm{~m}$ and terminates at level 42 . Column
C9 has a rectangular profile of $1.25 \times 1.25 \mathrm{~m}$ at levels 0 and 1 , an L-shaped profile of $1.95 \times 0.36 \mathrm{~m}$ and $1.265 \times 0.47 \mathrm{~m}$ from levels 2 to $23, a$ rectangular profile varying between $1.27 \times 0.61 \mathrm{~m}$ and $2.09 \times 0.56 \mathrm{~m}$ from levels 24 to 42 and a circular profile of $0.525 \mathrm{~m}$ diameter from levels 43 to 49. Walls $\mathrm{W} 1$ and $\mathrm{W} 2$ start as $0.6 \mathrm{~m}$ thick at level 0 , changing to $0.5 \mathrm{~m}$ thick at level 19 and $0.4 \mathrm{~m}$ thick at level 36 . W1 terminates at level 42 while W2 extends all the way to level 49.

\subsection{Fibre optic sensor system}

Each monitored location was instrumented with a pair of embedded fibre optic cables, entering the concrete at the ground floor level 0 (first floor level 1 in the case of column (9) and having sufficient length to be installed all the way to the top of the columns / walls (Figure 3.2).

One cable in each pair is a $5.2 \times 1.3 \mathrm{~mm}$ tightbuffer cable (referred to as strain cable, or '-Str') with four single-mode fibres and two steel reinforcement wires encased in a tightly bonded nylon jacket (Fujikura JBT-03813). The other cable is a $6 \mathrm{~mm}$-diameter loose-tube cable (referred to as temperature cable, or '-Tmp') with four singlemode fibres in a gel-filled inner tube, surrounded by reinforcing glass fibre strands and a thermoplastic jacket (Excel 205-300).

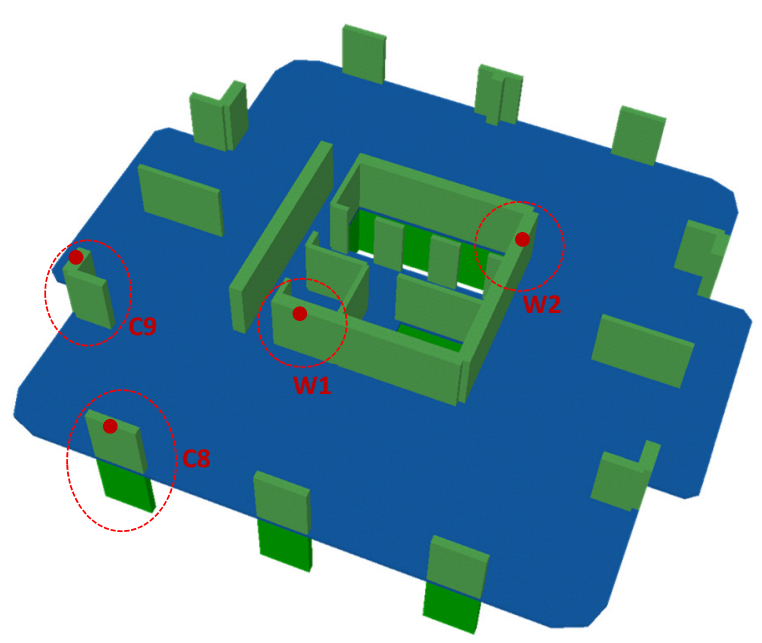

Figure 3.1. 3-D overview of the core and columns layout in a typical floor, showing the instrumented locations. 


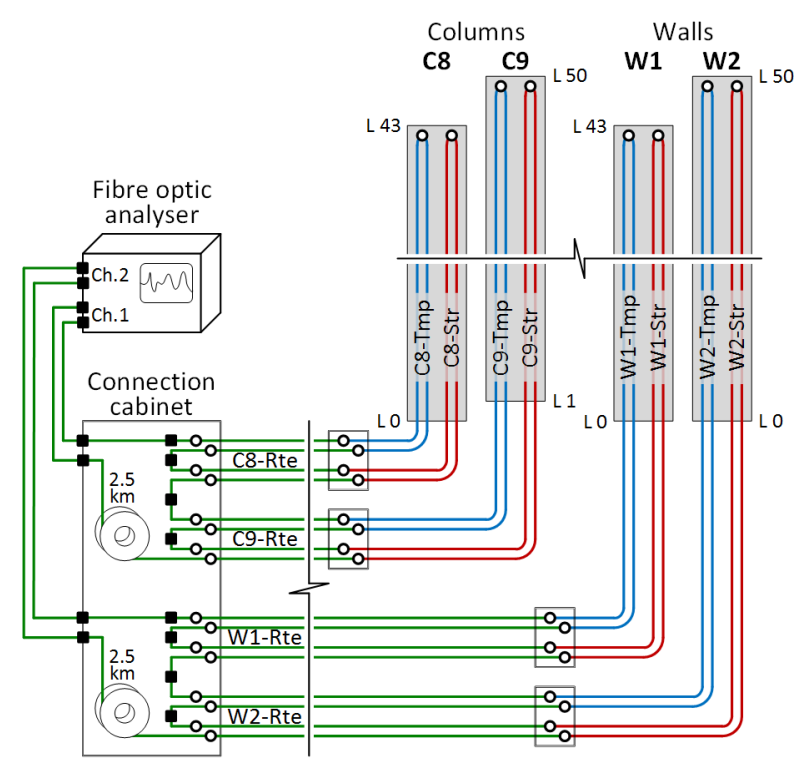

\begin{tabular}{|lll|}
\hline LEGEND: & o & Fusion splice \\
\hline Strain sensing optical fibre & Mechanical connector \\
Temperature sensing optical fibre & (e) & Fibre spool \\
\hline Routing optical fibre & & \\
\hline
\end{tabular}

Figure 3.2. Schematic diagram of the distributed fibre optic monitoring system at Principal Tower

The strain cable can be used to measure strain due to the combined thermal and mechanical actions, while the temperature cable can be used to measure temperature changes in the concrete. The temperature data can then be used to distinguish between strain induced by mechanical actions and strain induced by thermal expansion or contraction of the monitored elements [9].

Two of the four optical fibres within each cable are used for sensing. These fibres were fusion spliced together in advance, at the top end of the cable, such that each cable was made optically self-returning (Figure 3.2). The cables were then wound in enclosed plastic cable reels, which were integrated into the jumpform rig (Figure 3.3). This allowed the construction workers to unreel and install the fibre optic cables at each level, as the jumpform advanced upwards.

The cables were attached to a reinforcement bar on one side of the columns / walls using plastic cable ties. This ensured that the cables remained taut and in place until the concrete was poured. The strain cable was also pre-tensioned at each level. Since the instrumented columns and walls

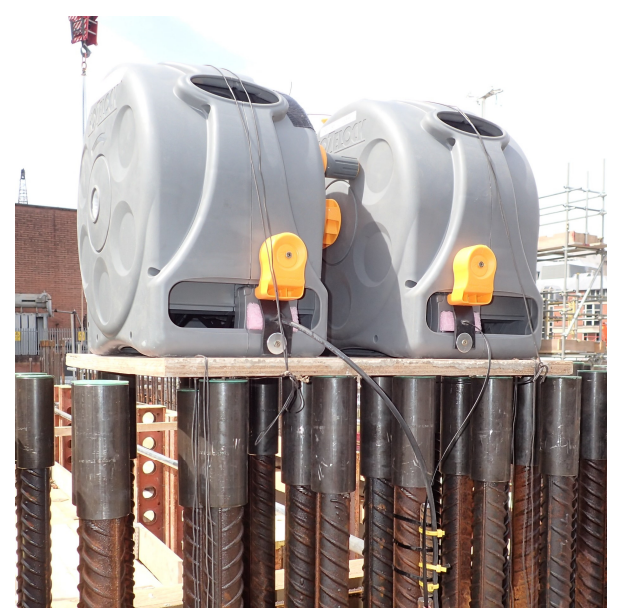

Figure 3.3. Fibre optic cable reels at one of the monitored walls, before they were integrated into the jumpform rig.

were not expected to carry any significant bending moment, measuring strain on one side of the elements was considered sufficient.

At ground level, the four sensing fibres (two per cable) exiting each column / wall were fusion spliced to individual fibres within a separate fourcore loose-tube cable (referred to as a routing cable, or '-Rte'). The routing cable coming from each column / wall was passed through a channel in the ground slab, and through a reinforced plastic tube, to terminate in a connection cabinet (Figure 3.4) inside a dedicated monitoring room within the site office. Here the 16 individual optical fibres (four from each of the four routing cables) were connected together with a

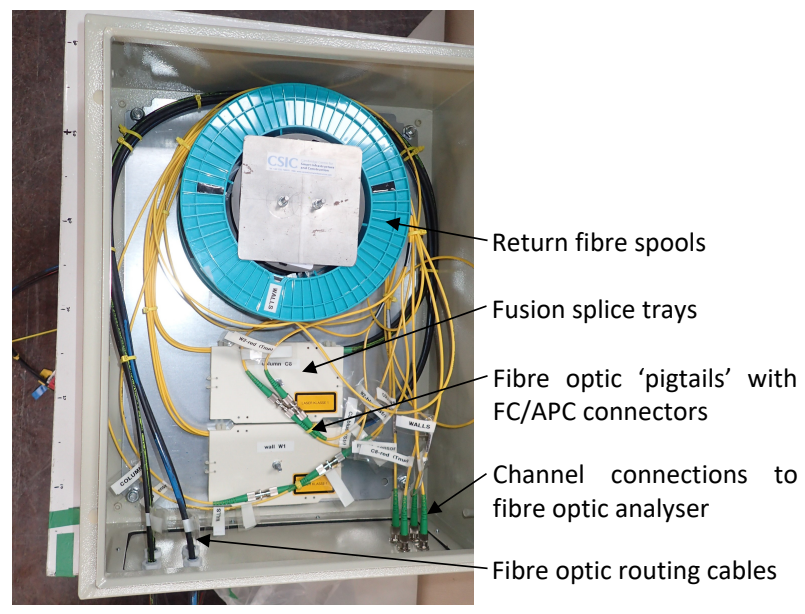

Figure 3.4. Fibre optic circuit connection cabinet. 
combination of fusion splices and FC/APC fibre optic connectors, to form two separate continuous circuits, one for the two columns and another for the two walls, as illustrated in Figure 3.2. The total approximate lengths of optical fibre in each circuit were $2.08 \mathrm{~km}$ and $1.93 \mathrm{~km}$ for the columns and walls, respectively.

\subsection{Fibre optic monitoring analyser}

The two ends of each circuit were connected to a dedicated channel of a two-channel BOTDA analyser (Omnisens DITEST STA-R), which is being kept permanently on site during the construction. Since the BOTDA technique used by this analyser can only take measurements over half of the optical fibre circuit, a $2.5 \mathrm{~km}$-long return fibre spool was connected between the end of each circuit and its analyser channel.

Distributed Brillouin sensing is based on the physical phenomenon of light scattering as it travels through an optical fibre. This is explained in detail in [10]. A distributed fibre optic analyser exploits this phenomenon by sending pulses of light through the fibre and detecting the amount of light scattered back towards it from every point along the fibre (known as backscattered light). The frequency spectrum of this backscattered light is made up of a number of distinct peaks, one of which is the Brillouin peak. The frequency of this peak shifts proportionally to the strain and temperature at the fibre where the light scattering occurred. Thus, by recording the Brillouin frequency of discrete points along the sensing fibre over time, one can derive the changes in strain and / or temperature within the fibre, and hence within the material in which it is embedded, at each of those points.

For the monitoring system at Principal Tower, the BOTDA analyser was set to take measurements at $0.20 \mathrm{~m}$ intervals along the optical fibre circuits, with a spatial resolution of $1 \mathrm{~m}$ and averaging over 3000 pulses. Measurements from each channel took around 8 minutes to complete and were repeated approximately every 30 minutes. The generated data files were stored in the analyser's internal memory and transferred automatically to an off-site secure backup server over an Internet connection.

\section{Monitoring data and discussion}

Monitoring of the columns and walls started on 3rd September 2016, as soon as the circuit connections were completed, and before any of the instrumented elements had been concreted. Since then, measurements have been taken continuously, with the exception of some periods during which monitoring was paused, either to carry out maintenance on the system, or due to unexpected software problems. The latter happened a number of times in the first three months, before it was eventually resolved.

Data were also lost whenever the sensing cables were accidentally damaged, until they could be repaired. This happened in C9, W1 and W2 during the first five months of operation. However, since the column and wall circuits are interrogated independently of each other by the analyser, if one circuit is damaged, measurements can still be taken from the other circuit.

The data acquired along the embedded sensing cables were post-processed to derive the change in concrete temperature, total axial strain, and mechanical axial strain (i.e. removing the effects of thermal movement, assuming a thermal expansion coefficient of $10 \mu \varepsilon /{ }^{\circ} \mathrm{C}$ ). The axial displacement was derived by integrating the strain values, starting from the lowest instrumented level of each element. Thus, the displacement calculated at each point of the element is relative to a datum at the bottom of level 0 for $\mathrm{C} 8, \mathrm{~W} 1$ and W2, and at the bottom of level 1 for $C 9$.

The average axial displacement of columns C8 and C9 recorded over a $1 \mathrm{~m}$ section at the middle of each level, up to level 4, is shown in Figure 4.1 and Figure 4.2 , respectively. The plots span until $14^{\text {th }}$ December 2016, by which time the slab and columns had been concreted up to level 5 . Negative displacement indicates shortening.

The displacement at each level is presented with respect to a baseline measurement. By default, the baseline for each level was the measurement recorded at around midnight immediately following the concreting of the element. The exceptions were $\mathrm{C} 8$ level 0 (12 days after concreting), C8 level 1 (3 days after concreting) and C9 level 4 ( 2 days after concreting). 
(a)

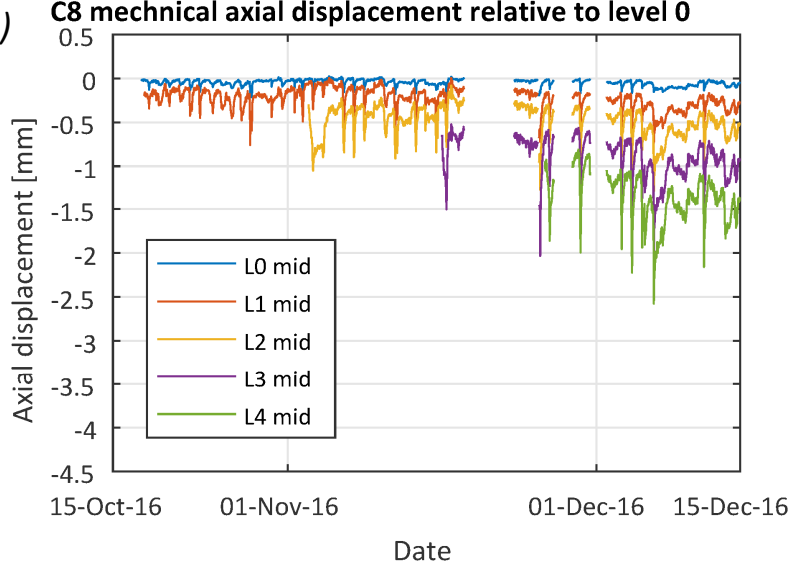

(b)

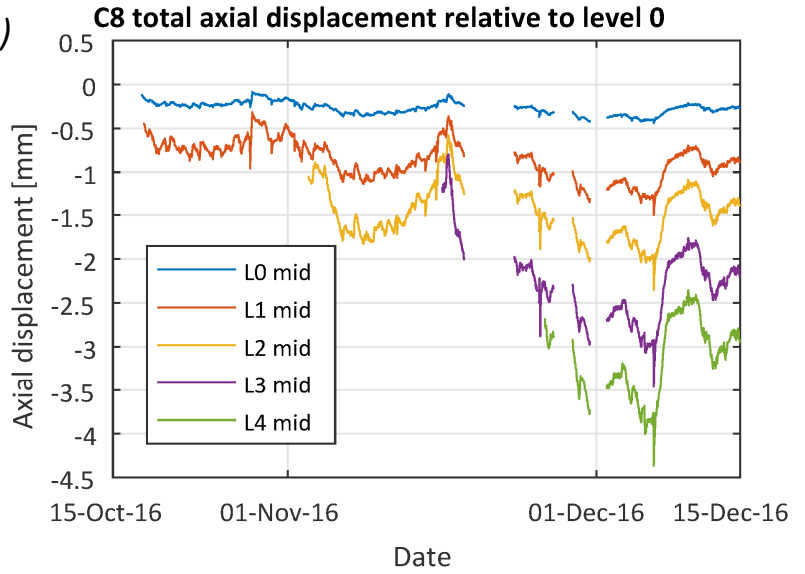

Figure 4.1. Axial displacement measured in column $\mathrm{C} 8$, due to (a) mechanical actions alone, and (b) combined thermal and mechanical actions.

(a)

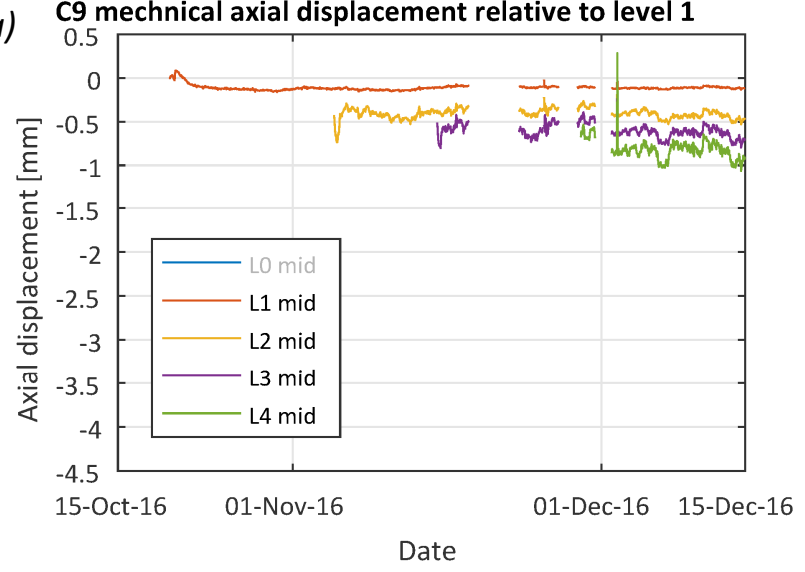

(b)

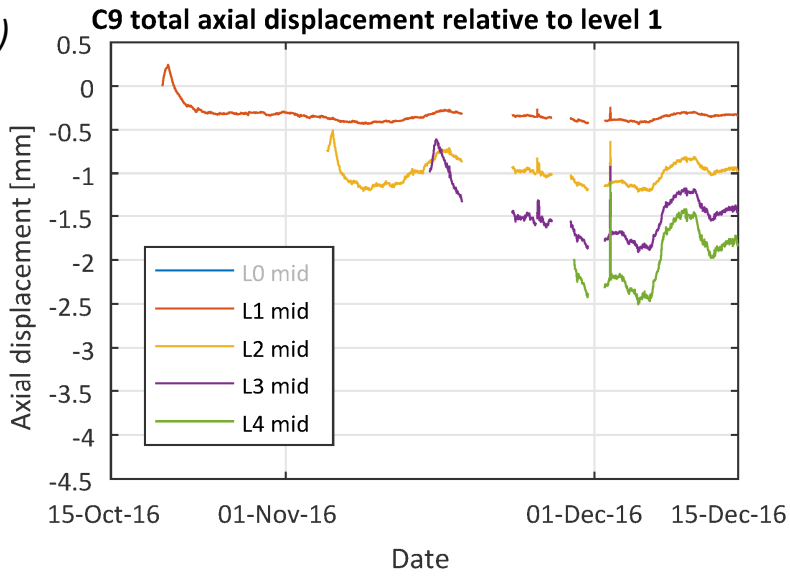

Figure 4.2. Axial displacement measured in column C9, due to (a) mechanical actions alone, and (b) combined thermal and mechanical actions.

The maximum column shortening recorded at level 4 due to mechanical actions was $2.9 \mathrm{~mm}$ for C8 (relative to level 0) and $1.2 \mathrm{~mm}$ for C9 (relative to level 1). At such an early stage in the construction, it is not surprising that the recorded column shortening was so low. Nonetheless, the monitoring system was able to measure these very small levels of deformation in detail.

When considering the combined effect of mechanical and thermal actions, the maximum recorded column shortening was $4.7 \mathrm{~mm}$ for $\mathrm{C} 8$ and $2.6 \mathrm{~mm}$ for $\mathrm{C} 9$. From these results, it is evident that, during the early stages of construction, when the building is lightly loaded, the axial deformation of the columns is dominated by their thermal expansion and contraction.
The plot showing mechanical displacement of $\mathrm{C} 8$ (Figure 4.1a) is characterised by several sharp peaks which occur on a diurnal basis on most days. Interestingly, the data acquired at the same time from C9 (Figure 4.2a) does not have these daily peaks. It is suspected that this is due to the instrumented face of $\mathrm{C} 8$ being directly exposed to the sun in the afternoon, whereas that of $C 9$ is constantly shaded. Consequently the temperature cable in $\mathrm{C} 8$ might be measuring the column's surface temperature rather than the average temperature over the cross-section. This would result in over-estimating the thermal expansion strain and therefore over-compensating the total strain data, leading to artificial compressive peaks in the mechanical strain and axial displacement (Figure 4.1a). This requires further investigation. 
Despite the limited amount of data recorded so $\mathrm{far}$, it is already clear that $\mathrm{C} 8$ is shortening more than C9. This is likely due to C8 having a smaller cross-sectional area and less reinforcement than C9. This differential shortening, which is expected to become more pronounced as the construction progresses, is an important consideration since the two columns are adjacent and only $6.2 \mathrm{~m}$ apart.

\section{Conclusions}

A distributed fibre optic strain sensor (DFOS) system is being installed in Principal Tower, a 50storey residential building in central London, to monitor the axial shortening of two columns and two core walls. Continuous fibre optic cables are embedded into these members as the construction progresses. At the same time, a Brillouin optical time domain analysis (BOTDA) analyser is constantly taking readings of strain and temperature from the embedded cables. These data are then processed to obtain a measure of axial displacement at any time, and at any location along the instrumented members.

The first few months of data acquired from the two columns have been presented and discussed in this paper. In addition to the continual data analysis as additional floors are constructed, future work will include a comparison of the measured and predicted shortening, a comparison of the column shortening with that of the walls, and an investigation into the influence of direct solar radiation on the temperature compensation of the data to remove the effects of thermal expansion.

\section{Acknowledgements}

The authors would like to acknowledge the contribution of Paul Fidler, Cedric Kechavarzi, Peter Knott, Jason Shardelow (CSIC); Diego PadillaPhilipps (WSP Group); Mason Brown (Multiplex Construction).

This research has been made possible through funding under EPSRC grant EP/N021614/1 and Innovate UK grant 920035.

The data presented in this paper can be obtained from https://doi.org/10.17863/CAM.7925

\section{References}

[1] Pan LB, Liu PC, Bakoss SL. Long-term shortening of concrete columns in tall buildings. Journal of Structural Engineering. 1993; 119(7): 2258-2262.

[2] Fintel M, Ghosh SK, lyengar H. Column shortening in tall buildings - Prediction and compensation. EB108.01D, Skokie, IL; 1986.

[3] Fintel M, Khan FR. Effects of column creep and shrinkage in tall structures - Prediction of inelastic column shortening. $\mathrm{ACl}$ Journal Proceedings. 1969; 66(12).

[4] Park SW, Choi SW, Park HS. Moving average correction method for compensation of differential column shortenings in high-rise buildings. The Structural Design of Tall and Special Buildings. 2013; 22(9): 718-728.

[5] Park HS. Optimal compensation of differential column shortening in high-rise buildings. The Structural Design of Tall and Special Buildings. 2003; 12(1): 49-66.

[6] Kim H, Cho S. Column shortening of concrete cores and composite columns in a tall building. The Structural Design of Tall and Special Buildings. 2005; 14(2):175-190.

[7] Choi SW, Kim Y, Kim JM, et al. Field monitoring of column shortenings in a high-rise building during construction. Sensors. 2013; 13(11): 14321-14338.

[8] Glisic B, Inaudi D, Lau JM, et al. Ten-year monitoring of high-rise building columns using long-gauge fiber optic sensors. Smart Materials and Structures. 2013; 22(5).

[9] Mohamad H. Temperature and strain sensing techniques using Brillouin optical time domain reflectometry. In: Proceedings of SPIE 8346, Smart Sensor Phenomena, Technology, Networks, and Systems Integration. San Diego, CA; 2012; 83461M.

[10] Kechavarzi C, Soga K, de Battista N, et al. Distributed Fibre Optic Strain Sensing for Monitoring Civil Infrastructure - A Practical Guide. London, UK: ICE Publishing; 2016. ISBN: 9780727760555 